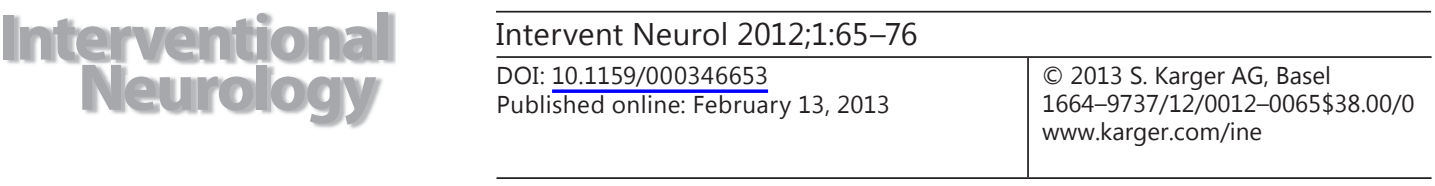

\title{
Review
}

\section{Multimodal CT versus MRI in Selecting Acute Stroke Patients for Endovascular Treatment}

\author{
Pablo García-Bermejo Carlos Castaño Antonio Dávalos \\ Department of Neurosciences, Hospital Universitari Germans Trias i Pujol, Badalona, Spain
}

\section{Key Words}

Acute ischemic stroke - Acute management of stroke $\cdot$ Acute stroke imaging $\cdot$ Multimodal imaging · Penumbra imaging · Endovascular treatment of stroke · Thrombectomy

\begin{abstract}
Arterial revascularization is the most effective therapy for acute stroke patients. Systemic thrombolysis with recombinant tissue plasminogen activator based on baseline non-contrast CT (NCCT) imaging criteria is the only treatment approved to date, which is limited by severe timing and medical restrictions, and a narrow efficacy. The simple imaging methodology required contrast with the current wide range of imaging modalities available, which permits the physician to rapidly obtain an estimation of the most relevant pathophysiological factors involved in an acute stroke, overcoming the limited capability of NCCT. Multimodal CT and MRI combine techniques that aim to depict the neurovasculature and the status of the brain parenchyma, including the presence of hemorrhage and infarction, as well as the viability of the cerebral ischemic areas involved. These additional imaging procedures have been explored in clinical studies and trials to guide intravenous thrombolysis in acute stroke patients beyond the currently established time windows, with inconclusive results. However, emergent endovascular materials and techniques are evolving, showing promising results with increasing rates of arterial recanalization with an acceptable safety profile, therefore becoming a potential alternative and complimentary treatment, although the current lack of a high level of evidence is limiting their use in routine clinical practice. Nevertheless, these advanced neuroimaging methods can be used to improve the selection of candidates for these novel invasive therapies and, moreover, to help the interventionist to design a faster and safer procedure. In this article, we review the basic aspects of both CT and MRI modalities and the state of the evidence of these imaging tools to guide endovascular therapy, as well as the advantages and disadvantages of each method.




\section{Introduction}

Stroke is a leading cause of death and disability worldwide, and, up to date, intravenous thrombolytic therapy with recombinant tissue plasminogen activator (rtPA) for acute ischemic stroke remains the only treatment available, which proved to be safe and effective in large clinical trials within $4.5 \mathrm{~h}$ of symptom onset [1]. This intervention is seriously limited by its short therapeutic window and its relatively low efficacy. Thus, only about $2-8 \%$ of patients who suffer an ischemic stroke in a region with appropriate facilities receive this treatment [2], mainly because of delayed arrival to the hospital or unknown symptom onset, while, probably due to its relatively low recanalization rate, 8 stroke patients must be treated with intravenous rtPA to achieve an additional patient with good functional outcome [1].

Endovascular therapy has emerged as a complementary or alternative tool for treating patients with acute ischemic stroke for whom intravenous rtPA is contraindicated or ineffective. During the last decades, different techniques have been developed, from the local administration of a fibrinolytic agent to the use of mechanical devices to disrupt or to remove the clot, increasing the rates of arterial recanalization with a theoretic reduction in the risk of symptomatic intracranial hemorrhage ( $\mathrm{sICH})$ and systemic bleeding. However, these procedures are relatively complex, time consuming and may carry an additional risk derived from its invasive nature. Moreover, its use has not been proven in clinical trials, and its cost-effectiveness remains to be determined. Therefore, it is warranted to improve the classical imaging selection method based on the simple non-contrast CT (NCCT) used in most of the intravenous rtPA and intra-arterial trials, in order to enhance the safety, efficacy and cost-effectiveness of these novel therapies.

Newer technological advances in stroke imaging offer a wider range of information that allows the physician to acutely visualize a precise snapshot of the physiological status of the stroke regarding the status of the neck and brain circulation, the extension and proportion of the irreversible and potentially salvageable ischemic brain tissue and in many cases even the etiology. This deeper additional information is being increasingly used to strategically overcome the strict restrictions of the current guidelines to apply a revascularization therapy, thus increasing the number of patients who may benefit from either intravenous thrombolysis or endovascular treatment.

We aim to review the general aspects of both modalities, CT and MRI, focusing on those particularities which may be of interest in order to select acute stroke patients for endovascular therapy and to plan the intra-arterial intervention.

\section{Neuroimaging of the Vasculature}

The most beneficial therapeutic intervention in acute ischemic stroke is the early restoration of the cerebral blood flow (CBF), which is closely related to the functional outcome. Distal intracranial arterial occlusions are more likely to recanalize than proximal ones in patients treated with intravenous rtPA. The recanalization rates of intravenous rtPA for proximal arterial occlusions range from less than $10 \%$ for internal carotid artery occlusion to $30 \%$ for proximal and $44 \%$ for distal middle cerebral artery occlusions [3]. However, endovascular therapy may be more successful for proximal occlusions, especially with the use of the novel stentrievers, which have overcome this limitation by reaching recanalization rates higher than 85\% [4]. Furthermore, several vasculature-related aspects regarding the location and burden of the clot, as well as the proximal and leptomeningeal circulation status, acquire tremendous relevance in order to plan an intervention. Moreover, imaging of the neck and 
brain vessels may allow an early identification of the etiology of the stroke, and therefore a fast initiation of targeted secondary prevention therapies. Both MR and CT allow a deep insight into the cerebral arterial irrigation system, with certain peculiarities.

\section{MR Angiography}

There are different techniques available to visualize the cervical and cerebral vasculature, 2D and 3D time-of-flight (TOF), multiple-overlapping thin-slab acquisition and contrastenhanced MR angiography (MRA). TOF-MRA is one of the most used techniques in the stroke assessment since contrast material injection is not required. The accuracy of this modality in the assessment of the intracranial vessel status is moderate [estimated sensitivity for detection of stenosis between 60 and 85\% (80-90\% for occlusions)] compared with CT angiography (CTA) and/or digital subtraction angiography (DSA), whereas the estimated sensitivity and specificity for the detection of extracranial carotid disease reach 93 and 88\%, respectively. Although its resolution for proximal lesions in the neck vessels and large intracranial arteries appears acceptable, distal branch occlusions are suspicious, probably due to motion artifacts at this level. Moreover, TOF-MRA can overrate the degree of an arterial stenosis, even its length, presenting instead a false vascular occlusion. This effect may be caused by saturation of slow flow. However, the use of gadolinium for contrast -enhanced MRA, which makes the acquisition faster, produces an angiographic effect which may overcome the low motion flow and turbulence artifacts, and therefore provides a higher accuracy for cervical arterial atherosclerotic disease than TOF-MRA [5]. Moreover, contrast-enhanced MRA after perfusion could even reproduce this effect in the intracranial vasculature, as it can depict arterial segments with low flow and avoid the overestimation of vascular stenosis and occlusion [6].

\section{CT Angiography}

This technique can be performed within a few minutes after the initial NCCT with an iodinated single-bolus contrast injection with a thin-slab and high-resolution multidetector spiral CT. Its axial CTA source images (SI) can be rapidly examined, even though postprocessing 3D computer reconstructions of the circle of Willis and extracranial cerebral arteries can be performed within a few minutes, providing images comparable to those obtained with DSA. The majority of the studies assessing the accuracy of CTA in the detection of arterial occlusion and stenosis of the extra- and intracranial vessels have reported higher sensitivity, specificity and positive predictive value than those for MRA. The sensitivity of CTA for the detection of intracranial occlusions is near 100\% (92-100\%) compared with the gold standard DSA, with a specificity of $82-100 \%$ and a positive predictive value of $91-100 \%$. The accuracy for extracranial lesion detection is also high, with similar percentages of sensitivity, specificity and positive predictive value according to several studies [5]. In addition, CTA offers an accurate estimation of the pial collateral circulation status, which predicts clinical and radiological outcome, and therefore facilitates a deeper comprehension of the pathophysiological process of the respective stroke to the attending physician [7].

Previous imaging of the supra-aortic and intracranial vessels offers a significant advantage in order to devise an individualized endovascular intervention. Previous knowledge of the supra-aortic artery caliber and status, besides the grade of the circle of Willis and pial collateralization, allows the interventionist to catheterize the afferent artery directly, avoiding unnecessary attempts at additional vessels and, consequently, saving precious time which can be vital in these patients. Moreover, the previous awareness of the extent of the clot beyond its proximal location helps to position the microcatheter with a higher confidence beyond its distal tip, therefore avoiding unnecessary further displacement and allowing a deployment of the device with a higher precision to trap the entire thrombus in the case of a mechanical thrombectomy. For example, in patients with an occlusion of the top of the basilar 
artery, CTA can display in which of the P1 segments the clot is more extended, hence indicating where to place the distal tip of the stent to increase the chance of successful recanalization (fig. 1).

\section{Neuroimaging of the Parenchyma}

\section{Hemorrhage}

The first diagnostic step in the hyperacute phase of a stroke patient is to confirm or exclude an ICH. The confirmation of a brain bleeding leads to a very different management compared with a normal or an ischemic brain lesion.

NCCT is highly sensitive for the detection of brain hemorrhage and is also considered the most cost-effective strategy when performed in the hyperacute phase [5]. The concerns of the past regarding the accuracy of MRI to detect ICH in the acute phase of stroke have been overcome by the results of several recent studies reporting a similar accuracy to NCCT for the detection of acute ICH in patients presenting with focal neurological symptoms. Moreover, MRI may be even more accurate than NCCT in the detection of subacute and chronic ICH and hemorrhagic transformation [5]. Hence, in order to select a stroke patient for endovascular treatment, both techniques appear to be equal.

One of the most feared complications of revascularization therapies is the occurrence of sICH. Factors associated with a higher risk of sICH after endovascular treatment appear to be the same as those related to the treatment with intravenous rtPA (e.g. stroke severity according to the baseline NIHSS score, the extent of early CT changes, time to treatment and delayed revascularization, lytic dose usage, medical conditions such as thrombocytopenia, uncontrolled hyperglycemia and hypertension, use of anticoagulants and antiplatelets, and atrial fibrillation), while other factors, such as moderate or severe leukoaraiosis in the deep white matter, may be associated with a higher risk of sICH after mechanical thrombectomy [8].

The DEFUSE-2 (Diffusion and Perfusion Imaging Evaluation for Understanding Stroke) is a multicenter, prospective cohort study of 104 acute stroke patients who were planned to undergo endovascular therapy within $12 \mathrm{~h}$ of symptom onset with a baseline and follow-up multimodal MRI performed. A substudy suggested that patients with a malignant profile, defined by volumes of diffusion-weighted imaging (DWI) $\geq 70 \mathrm{ml}$ and/or perfusion-weighted imaging (PWI; $\left.\mathrm{T}_{\max }>10 \mathrm{~s}\right) \geq 100 \mathrm{ml}$, who experience reperfusion $(>50 \%$ reduction in the PWI volume) present a higher risk of hemorrhagic transformation than those with a nonmalignant profile (63 vs. $20 \%$ PH1 or PH2, respectively, $\mathrm{p}=0.02$ ) [9].

\section{Imaging the Ischemic Brain Tissue Viability}

Most of the efforts in stroke neuroimaging research within the last decades have focused on the visualization of the ischemic core and penumbra. Thus, besides revealing the extent of the irreversible ischemic brain tissue (infarct core), the goal of multimodal stroke imaging is to disclose the area of hypoperfused parenchyma potentially salvageable that is functionally impaired but structurally intact and is destined to undergo infarction in the absence of reperfusion (penumbra), assuming the hypothesis that those patients with a significant core/ penumbra mismatch may be the target for revascularization therapies.

\section{Evaluation of Irreversible Ischemia}

Detection of early irreversible ischemia after excluding brain hemorrhage, besides the exclusion of conditions that may mimic stroke, is the next mandatory step in the management 
García-Bermejo et al.: Multimodal CT versus MRI in Selecting Acute Stroke Patients for Endovascular Treatment
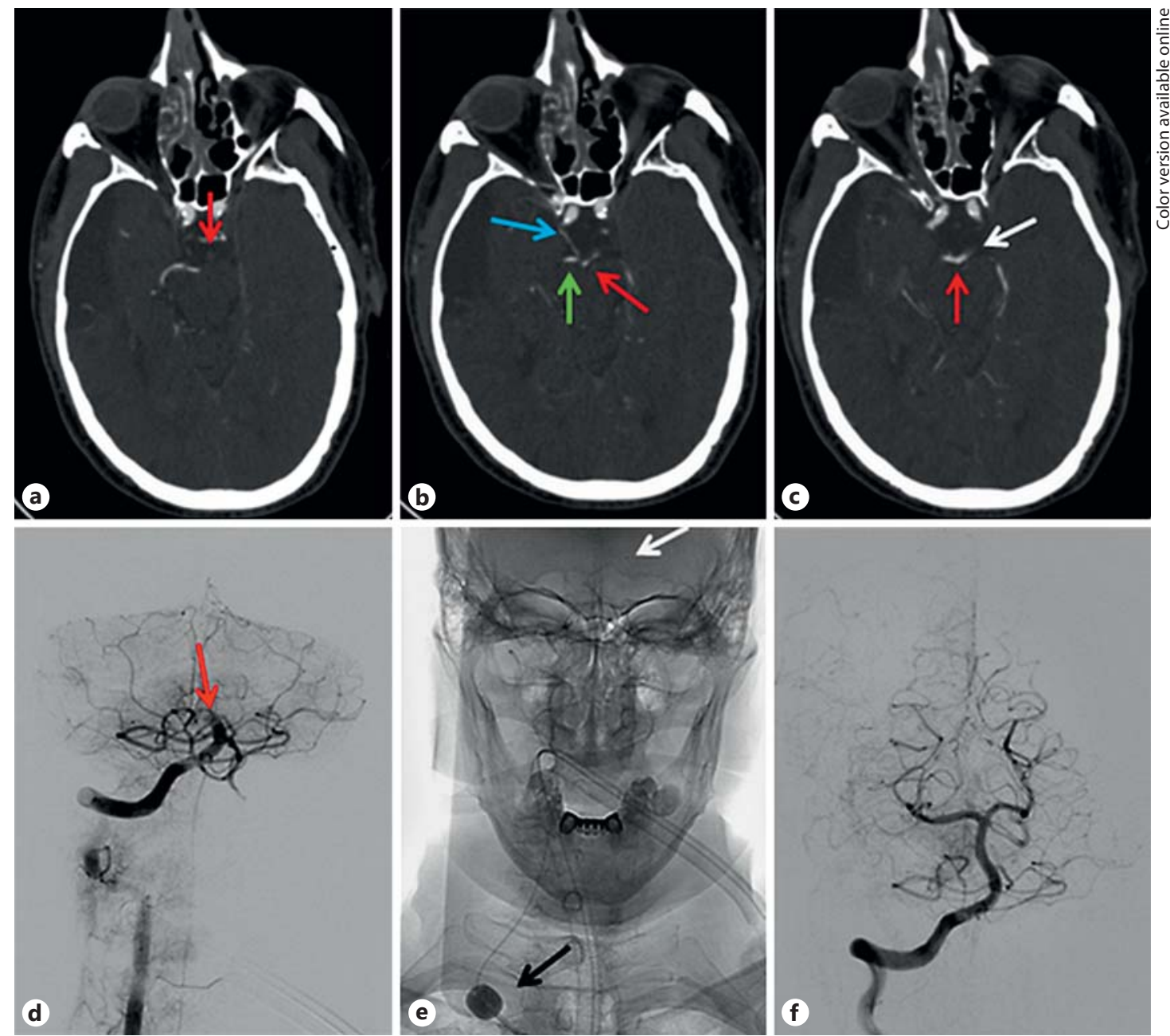

Fig. 1. CTA-SI before the procedure shows basilar artery occlusion (a, red arrow) with a patent right posterior communicating artery (b, blue arrow) that fills the right posterior cerebral artery (PCA; b, green arrow) and the 'top' of the basilar artery (b, c, red arrows), and an extent of the thrombus to the P1 segment of the left PCA (c, white arrow). Periprocedural DSA run confirms basilar artery occlusion (d, red arrow). Previous CTA information allows direct placement of the stentriever over the P1 segment of the left PCA where the distal tip of the clot is located (e, white arrow). The stent is retrieved under aspiration and occlusion of the right subclavian artery with a balloon guide catheter (e, black arrow), reaching complete recanalization and achieving a thrombolysis in cerebral infarction score 3 (f). Colors refer to the online version only.

of a stroke. The degree of extension of an early infarction is strongly related with the functional outcome [10], therefore patients with large baseline infarcts should be excluded from treatment due to its lack of benefit and higher risk of treatment-related adverse events.

\section{MRI Studies}

The most accurate sequence to detect early cerebral ischemia is DWI. Swelling of the ischemic brain cells due to a failure in the energy-dependent membrane pumps translates into a modification of the intra- to extracellular volume fractions that is detected by the DWI sequence. Vasogenic edema can also shine through DWI due to a component of a T2 effect, and therefore it is difficult to be distinguished from cytotoxic ischemic swelling. This problem 
can be solved by use of the apparent diffusion coefficient, which shows a reduced hypointense signal in infarcted tissues due to decreased water diffusion and increased hyperintense signal in vasogenic edema due to increased water diffusion [5].

MRI-DWI is highly sensitive and specific for the detection of early infarction and has become the best technique to detect acute ischemia [11], with a strong correlation with clinical outcome. There has been some concern about the reversibility of the acute DWI lesions, and the likelihood of inclusion of 'tissue at risk' inside these areas. Although 'DWI normalization' appears to be a relatively common phenomenon (6-20\%) in several studies, the reversal of the lesion is commonly associated with early tissue reperfusion and usually affects low volumes (mean 2.7-16 ml) [12]. Moreover, the clinical impact of this phenomenon remains unclear. Any related significant clinical improvement seems more likely due to the accompanying penumbra salvaged, which is associated with the tissue reperfusion, as the normalized DWI volumes involved are estimated to be very low.

Fluid-attenuated inversion recovery (FLAIR) is a T2-weighted sequence commonly used in multiparametric stroke MRI protocols; it is highly sensitive for subacute ischemic brain lesions but unable to detect ischemia within the first few hours. Visibility of ischemic lesions on FLAIR is time dependent, increases during the first $6 \mathrm{~h}$ of onset and reaches nearly $100 \%$ thereafter. Thus, DWI-FLAIR mismatch has the potential to become a 'tissue-clock' marker of the time of evolution of symptoms, which is useful to guide thrombolytic therapy when its origin remains unclear. A large multicenter prospective study showed a moderate specificity $(78 \%)$ and positive predictive value (83\%) to identify patients within $4.5 \mathrm{~h}$ of symptom onset [13]. However, its role in the selection of patients for endovascular therapy remains unexplored, as well as its capacity to predict hemorrhagic transformation.

CT Studies

NCCT has been widely used for the selection of candidates for intravenous thrombolysis despite its low sensitivity for the detection of early brain infarction, which has been estimated in a systematic review as $61 \%$ (SD, $\pm 21 \%$ ) within $6 \mathrm{~h}$ of stroke onset. This rate of detection may be increased by the use of the ASPECTS (Alberta Stroke Program Early CT Score) systematic analysis, besides adapting the window level to enhance the abnormal signs [5]. Moreover, these signs may have a different histological correlation: whereas hypoattenuation may represent irreversible ischemia, areas of swelling may represent potentially reversible ischemia [14].

The use of contrast media in multidetector spiral CT scanners may represent an advantage in the detection of early ischemia. CTA-SI enables to estimate parenchymal perfusion by enhancing the microvasculature with the contrast medium filling during its performance. Thus, findings with the first-generation scanners present a good correlation with DWI lesions and are better predictors of clinical and radiological outcomes than changes in NCCT, although new-generation scans, with faster acquisition protocols, may overestimate the size of the baseline infarct when the acquisition is not correctly performed [15].

An additional bolus of contrast media, with appropriate software to analyze the kinetics of the passage of dye through the brain parenchyma, allows the acquisition of CT perfusion (CTP) images. Although this technique may be more accurate than NCCT and CTA-SI for the detection of ischemia, there is no consensus in the literature regarding the thresholds and the best CTP map to define irreversible versus potentially reversible ischemia, probably due to the wide range of variability among CT scans and postprocessing software. CTP cerebral blood volume (CBV) maps have been classically chosen to estimate the infarcted core, while $\mathrm{CBF}$ and mean transit time (MTT) maps correlate better with the penumbra tissue under appropriate thresholds [16]. More recent studies are questioning the classical assessment of core and penumbra by CTP, suggesting that CBV core lesions may underestimate the final 
infarct size, whereas CBF may be more accurate than CBV to define the ischemic core [17]. Moreover, additional information can be obtained from CTP. CTP-SI provides a dynamic overview of CBF and allows a direct estimation of the penumbra and core [5].

\section{Evaluation of the Potentially Reversible Ischemia}

There are two main methodologies to assess the ischemic penumbra. Techniques based on the use of a diffusible tracer (positron emission tomography, and single photon emission and xenon CT) are considered reference procedures, although they are not operational. The most used methodology in clinical practice [MR perfusion (MRP) and CTP] comprises the use of a bolus injection of contrast medium, followed by dynamic assessment of the passage of the dye through the brain areas and a postprocessing computerized method. The regional analysis of the amount of blood in certain areas of the brain provides CBV maps, whereas analysis of the time provides maps of CBF, MTT, time to peak and $\mathrm{T}_{\text {max }}$. Both CTP and MRP methods aim to define the upper and lower limits of the penumbra. A simple model of diffusion-perfusion mismatch was initially proposed to provide an estimation of the penumbra. The difference between the area delimited by the DWI (estimated irreversible core) and the perfusion-demarked ischemic region (mismatch area) would correspond with the brain tissue at risk of infarction or salvageable if reperfusion occurs (estimated penumbra).

\section{MRI-PWI Studies}

Most of MRP studies are performed with the first-pass bolus technique, using an intravenous bolus of gadolinium. CBF can also be studied with the use of arterial spin labeling sequences, which magnetically label the blood entering the brain without the use of any contrast. Perfusion-defect areas are very sensitive detecting ischemia, predict infarct growth and may correlate better than DWI lesions with the clinical and radiological outcome in the absence of reperfusion, whereas patients without mismatch did not experience subsequent growth of the infarct. On the other hand, the DEFUSE study demonstrated that patients with a significant baseline DWI-PWI mismatch treated with intravenous rtPA within 3-6 h who experience early reperfusion present better outcomes than those with no reperfusion, whereas patients with no baseline mismatch did not benefit from reperfusion. Despite these facts, perfusion deficits do not predict how much the initial infarct increases in cases with no recanalization, which makes it difficult to reliably establish definite PWI thresholds to discriminate penumbra from benign oligemia, and leads to an overestimation of the real size of the penumbra by the most commonly used PWI parameters $[5,18]$.

\section{CT Studies}

The accuracy of CTP maps in the identification of cerebral penumbra in acute stroke patients has been validated in several studies in comparison with xenon CT, positron emission tomography and MRP, and may be equal, or even superior, to MRI PWI/DWI, although specific situations such as posterior circulation strokes and lacunar infarcts limit this technique [5]. Distinction of the irreversible infarct from the penumbra with CTP maps is based on the cerebral vascular autoregulation concept. MTT maps are very sensitive to cerebral ischemia and may be delayed in both conditions. In the core, the cerebral vasoreactivity is impaired, thus CBV is low and CBF should be extra low as well, whereas in the penumbra, as brain vessel reactivity is still preserved, CBV should be normal or even increased, while CBF should be slightly decreased to a certain threshold $[5,16]$.

Despite profuse research efforts, up to date, upper and lower CT and MRI penumbral thresholds have not been accurately established. 


\section{\begin{tabular}{l|l}
\hline DOI: $10.1159 / 000346653$ & ( 2013 S. Karger AG, Basel
\end{tabular} www.karger.com/ine \\ García-Bermejo et al.: Multimodal CT versus MRI in Selecting Acute Stroke Patients for Endovascular Treatment \\ Multimodal Imaging-Based Selection of Acute Stroke Patients for Endovascular Treatment}

Data supporting the use of endovascular treatment in acute ischemic stroke are still of low quality and its impact on survival and functional outcome remains uncertain. There is only one randomized clinical trial that demonstrated improved functional outcome with intra-arterial thrombolysis (IAT) [PROACT-II (Prolyse in Acute Cerebral Thromboembolism)]. A recent systematic review showed that most of the data from interventional studies come from uncontrolled studies, with only 18 prospective studies and 3 with blinded assessment of outcome until November 2010 [19]. However, novel stentrievers are showing promising results $[4,20]$ and may be even superior to previous devices, as shown in a recent nonpublished trial: SWIFT (Solitaire with the Intention for Thrombectomy) [21].

The imaging criteria for the selection of patients for intra-arterial treatment in most of the interventional trials have been based on standard NCCT findings [ECASS (European Cooperative Acute Stroke Study) criteria]. Nonetheless, most of the nonrandomized clinical series exploring the use of endovascular treatment have used CTP, while multimodal MRI have been used to explore mainly intravenous rtPA in this setting.

\section{Multimodal CT Experience}

Abou-Chebl [22] prospectively studied 55 patients treated with intra-arterial tPA plus rescue Merci clot retrieval who were selected with multimodal CT and presented large vessel occlusion with mismatch, and compared patients treated within $(\mathrm{n}=34)$ and beyond $6 \mathrm{~h}(\mathrm{n}=$ 21). Successful recanalization $(82.8 \%<6$ h vs. $85.7 \%>6 \mathrm{~h}, \mathrm{p}=1.0)$, $\operatorname{sICH}(8.8 \%<6 \mathrm{~h}$ vs. $9.1 \%$ $>6 \mathrm{~h}, \mathrm{p}=1.0)$ and functional outcome rates $(41.2 \%<6 \mathrm{~h}$ vs. $42.9 \%>6 \mathrm{~h}, \mathrm{p}=0.902)$ were similar. In addition, in a prospective multicenter study of 237 acute stroke patients selected with either multimodal CT $(n=120,61 \%)$, MRI $(n=53,26 \%)$ or both $(n=26,13 \%)$ and anterior circulation occlusion beyond $8 \mathrm{~h}$ from symptom onset who were treated with endovascular therapy, Jovin et al. [23] showed favorable rates of recanalization (73.84\%), sICH (8.86\%), functional 3-month outcome (modified Rankin scale $\leq 2: 45 \%$ ) and mortality (21.5\%). However, in a retrospective study comparing 69 CTP-guided versus 127 NCCT timeguided acute ischemic stroke patients (onset to treatment time: $308 \mathrm{vs.} 301 \mathrm{~min}, \mathrm{p}=0.08$ ) treated with intra-arterial therapy, Hassan et al. [24] reported similar rates of recanalization ( 88 vs. $81 \%, p=0.52$ ) and good functional outcome (32 vs. $33 \%, p=0.9$ ) between the two groups. Thus, these results suggest that multimodal CT-guided endovascular treatment may be as safe and effective in delayed as in nondelayed time windows. However, whether multimodal CT-assisted selection offers an advantage over standard NCCT remains unclear.

\section{Multimodal MR Experience}

The DWI-PWI mismatch criteria have been studied in several multicenter studies and randomized clinical trials reporting that there is still no confirmation that patients with mismatch improve with intravenous thrombolysis [25]. According to the positive results of the DEFUSE study mentioned above, the DIAS (Desmoteplase in Acute Ischemic Stroke) and DEDAS (Dose Escalation of Desmoteplase for Acute Ischemic Stroke) trials showed promising results in patients selected according to the mismatch hypothesis and treated with intravenous desmoteplase within delayed time windows. Nevertheless, the EPITHET (Echoplanar Imaging Thrombolytic Evaluation Trial) failed to demonstrate a difference between patients with and without mismatch in the degree of infarct growth in both groups of patients treated with intravenous rtPA and placebo. In addition, the DIAS II trial failed to demonstrate a benefit of intravenous desmoteplase treatment within 3-9 h in patients selected with $20 \%$ estimated penumbra with either CT or MRI $[5,18,25]$. 
García-Bermejo et al.: Multimodal CT versus MRI in Selecting Acute Stroke Patients for Endovascular Treatment

Table 1. Advantages and disadvantages of multimodal CT and MRI modalities

\begin{tabular}{|c|c|c|c|c|c|}
\hline & & General facts & Imaging early infarction & Imaging the vasculature & Imaging the penumbra \\
\hline \multirow[t]{2}{*}{ CT } & Advantages & Available, rapid, cheap & $\begin{array}{l}\text { Validated in clinical i.v. } \\
\text { rtPA trials and IAT } \\
\text { (PROACT II); } \\
\text { Cost-effectiveness }\end{array}$ & $\begin{array}{l}\text { Highly accurate } \\
\text { (near } 100 \% \text { ); } \\
\text { Less motion artifacts }\end{array}$ & $\begin{array}{l}\text { Experience in multi- } \\
\text { centric clinical studies } \\
\text { supports its use for IAT }\end{array}$ \\
\hline & Disadvantages & $\begin{array}{l}\text { Radiation exposure, } \\
\text { contrast agent } \\
\text { limitations }\end{array}$ & $\begin{array}{l}\text { Moderate accuracy and } \\
\text { interobserver reliability; } \\
\text { Uncertainty beyond } 4.5 \mathrm{~h}\end{array}$ & $\begin{array}{l}\text { Radiation exposure and } \\
\text { iodinated contrast } \\
\text { enhancement needed }\end{array}$ & $\begin{array}{l}\text { Lack of standardization } \\
\text { of methods, and post- } \\
\text { processing software; } \\
\text { Limited in posterior } \\
\text { circulation strokes }\end{array}$ \\
\hline \multirow[t]{2}{*}{ MRI } & Advantages & $\begin{array}{l}\text { No iodinated media } \\
\text { injection is needed }\end{array}$ & $\begin{array}{l}\text { Highly sensitive and } \\
\text { accurate }\end{array}$ & $\begin{array}{l}\text { Indicated in patients } \\
\text { with renal impairment }\end{array}$ & $\begin{array}{l}\text { Evidence supporting its } \\
\text { use with i.v. rtPA within } \\
6 \text { h (large studies, } \\
\text { DEFUSE) }\end{array}$ \\
\hline & Disadvantages & $\begin{array}{l}\text { Contraindications: metal } \\
\text { implants, claustrophobia, } \\
\text { unstable patients; } \\
\text { Less available and more } \\
\text { expensive, and specially } \\
\text { trained staff is needed; } \\
\text { Longer time may be needed }\end{array}$ & $\begin{array}{l}\text { Requires use of the } \\
\text { apparent diffusion } \\
\text { coefficient to confirm } \\
\text { cytotoxic vs. vasogenic } \\
\text { edema }\end{array}$ & Limited accuracy & $\begin{array}{l}\text { Lack of studies with } \\
\text { endovascular therapy; } \\
\text { Overestimation of } \\
\text { the penumbra; } \\
\text { Mismatch of } 20 \% \text { is } \\
\text { arbitrary }\end{array}$ \\
\hline
\end{tabular}

Specific studies exploring the use of the MRI mismatch criteria to select patients for endovascular treatment are scarce in the literature. The recent DEFUSE 2 study found that among patients selected for IAT with a baseline multimodal MRI and subsequent revascularization, those with MRI target mismatch before treatment [defined as DWI $<70 \mathrm{ml}+\mathrm{PWI}\left(\mathrm{T}_{\max } 10 \mathrm{~s}\right)$ $\left.<100 \mathrm{ml}+\mathrm{DWI} / \mathrm{PWI}\left(\mathrm{T}_{\max } 6 \mathrm{~s}\right)>1.8\right]$ who had early reperfusion $(>50 \%$ reduction in the PWI volume) after IAT had a higher likelihood of good clinical response (odds ratio 8.8 and $95 \%$ confidence interval 2.7-29.0), whereas patients without mismatch did not benefit from reperfusion (odds ratio 0.2 and 95\% confidence interval 0.0-1.6) [26].

However, the mismatch hypothesis carries several basic limitations. The common DWI-PWI mismatch criterion of $20 \%$ is arbitrary and has not been validated, probably due to its low specificity as most of the patients with a proximal vessel occlusion present with such a mismatch [27]. In addition, the visual assessment of the mismatch is associated with a significant degree of measurement error. Thus, the $20 \%$ mismatch criterion is under revision. A recent post hoc analysis of pooled DIAS, DEDAS and DIAS 2 data suggested that intravenous desmoteplase may be beneficial in patients with a higher baseline DWI-PWI mismatch $(\geq 60$ $\mathrm{ml}$ ) and ineffective in those with lower mismatch volumes [28]. Moreover, there is still no consensus on which PWI parameter (MTT, time to peak and $\mathrm{T}_{\max }$ ) and threshold should be used to accurately define the ischemic penumbra, which is overestimated in most of the cases [18].

However, the volume of the pretreatment DWI lesion appears to be a better predictor of clinical outcome in patients with successful early reperfusion than the PWI volume. In a study of 38 patients with internal carotid artery or middle cerebral artery occlusion treated with intravenous rtPA, and explored with baseline and follow-up multimodal MRI, baseline DWI volume $>89 \mathrm{ml}$ was found to be the only predictor of early neurological deterioration (sensitivity $85.7 \%$ and specificity 95.7\%) [29]. Consequently, a study of 34 patients treated with IAT who underwent previous multimodal MRI showed that all patients with initial DWI 
lesion $>70 \mathrm{ml}$ presented a poor outcome despite a recanalization rate of $50 \%$, whereas $64 \%$ of patients with initial DWI lesion volume $<70 \mathrm{ml}$ reached a good long-term clinical outcome ( $\mathrm{p}<0.008$ ), even with significantly less infarct growth (118 vs. $18 \mathrm{ml}, \mathrm{p}<0.03$ ) [30]. Other studies have confirmed these findings, suggesting a DWI lesion volume $>70 \mathrm{ml}$ as the threshold indicating a poor outcome, which is lower than the commonly used rule of the one third of the territory of the middle cerebral artery $(\sim 100 \mathrm{ml})$ [19]. Therefore, baseline DWI lesion volume in patients with proximal artery occlusion, rather than DWI-PWI mismatch, appears to be a better predictor of treatment response. Thus, patients with proximal artery occlusion and a small DWI lesion could be a better target population to undergo endovascular therapy.

\section{Advantages and Disadvantages of These Techniques}

Both multimodal neuroimaging modalities, CT and MRI, carry the same general limitations and advantages compared with those related to the standard acute stroke management. MRI may be limited by its inherent contraindications (e.g. implanted devices and claustrophobia) in about $10 \%$ of patients and by stroke-related issues (e.g. diminished level of consciousness, vomiting, agitation and hemodynamic compromise) in another $10 \%$ of cases; there are also additional barriers related to its availability and the necessity of trained staff besides the concern of imaging-related delays. MRI has been demonstrated to be feasible in this framework, and time limitations may be solved with gained experience, as far as to equal CT door-to-needle times $[6,19]$. On the other hand, CTP is more often available, faster and cheap. Moreover, CTP has fewer contraindications than MRI and patients tolerate it better. Even though, older CT generations have lesser brain coverage than MRI, and repeated multimodal CT exposure raises concerns about radiation exposure.

These features combined with the advantages and disadvantages of each CT and MR modality are summarized in table 1 .

\section{Acknowledgments}

Special thanks go to Dr. Josep Munuera for his kind assistance in the revision of this article.

\section{Disclosure Statement}

Authors report no conflict of interest.

\section{References}

1 Hacke W, Kaste M, Bluhmki E, Brozman M, Dávalos A, Guidetti D, Larrue V, Lees KR, Medeghri Z, Machnig T, Schneider D, von Kummer R, Wahlgren N, Toni D: Thrombolysis with alteplase 3 to 4.5 hours after acute ischemic stroke. N Engl J Med 2008;359:1317-1329.

2 Qureshi AI, Kirmani JF, Saved MA, Safdar A, Ahmed S, Ferguson R, Hershey LA, Qazi KJ: Time to hospital arrival, use of thrombolytics, and in-hospital outcomes in ischemic stroke. Neurology 2005;64:2115-2120.

3 Rha JH, Saver JL: The impact of recanalization on ischemic stroke outcome: a metaanalysis. Stroke 2007;38: 967-973.

4 Dávalos A, Pereira VM, Chapot R, Bonafé A, Andersson T, Gralla J, Solitaire Group: Retrospective multicenter study of Solitaire FR for revascularization in the treatment of acute ischemic stroke. Stroke 2012;43:2699_ 2705. 


\begin{tabular}{l|l}
\hline Intervent Neurol 2012;1:65-76 \\
\hline DOI: $10.1159 / 000346653$ & $\begin{array}{l}\text { @ 2013 S. Karger AG, Basel } \\
\text { www.karger.com/ine }\end{array}$ \\
\hline
\end{tabular}

García-Bermejo et al.: Multimodal CT versus MRI in Selecting Acute Stroke Patients for Endovascular Treatment

- 5 Latchaw RE, Alberts MJ, Lev MH, Connors JJ, Harbaugh RE, Higashida RT, Hobson R, Kidwell CS, Koroshetz WJ, Mathews V, Villablanca P, Warach S, Walters B: Recommendations for imaging of acute ischemic stroke: a scientific statement from the American Heart Association. Stroke 2009;40:3646-3678.

- 6 Pedraza S, Silva Y, Mendez J, Inaraja L, Vera J, Serena J, Dávalos A: Comparison of preperfusion and postperfusion magnetic resonance angiography in acute stroke. Stroke 2004;35:2015-2010.

7 Tan JC, Dillon WP, Liu S, Adler F, Smith WS, Wintermark M: Systematic comparison of perfusion-CT and CT-angiography in acute stroke patients. Ann Neurol 2007;61:533-543.

8 Shi ZS, Loh Y, Liebeskind DS, Saver JL, Gonzalez NR, Tateshima S, Jahan R, Feng L, Vespa PM, Starkman S, Salamon N, Villablance JP, Ali LK, Ovbiagele B, Kim D, Viñuela F, Duckwiler GR: Leukoaraiosis predicts parenchymal hematoma after mechanical thrombectomy in acute ischemic stroke. Stroke 2012;43:1806-1811.

9 Mlynash M, Lansberg MG, Straka M, Kemp S, Wechsler LR, Jovin TG, Wilder MJ, Lutsep HL, Czartoski T, Bernstein RA, Chang CW, Warach S, Fazekas F, Thai D, Inoue M, Tipirneni A, Hamilton SA, Zaharchuk G, Marks MP, Bammer R, Albers GW: The malignant MRI profile: implications for endovascular therapy. International Stroke Conference, New Orleans, La., USA, 2012.

10 Wardlaw JM, Mielke 0: Early signs of brain infarction at CT: observer reliability and outcome after thrombolytic treatment - systematic review. Radiology 2005;235:444-453.

11 Fiebach JB, Schellinger PD, Jansen O, Meyer M, Wilde P, Bender J, Schramm P, Jüttler E, Oehler J, Hartmann M, Hähnel S, Knauth M, Hacke W, Sartor K: CT and diffusion-weighted MR imaging in randomized order: diffusionweighted imaging results in higher accuracy and lower interrater variability in the diagnosis of hyperacute ischemic stroke. Stroke 2002;33:2206-2210.

12 Chemmanam T, Campbell BC, Christensen S, Nagakane Y, Desmond PM, Bladin CF, Parsons MW, Levi CR, Barber PA, Donnan GA, Davis SM: Ischemic diffusion lesion reversal is uncommon and rarely alters perfusiondiffusion mismatch. Neurology 2010;75:1040-1047.

13 Thomalla G, Cheng B, Ebinger M, Hao Q, Tourdias T, Wu O, Kim JS, Breuer L, Singer OC, Warach S, Christensen S, Treszl A, Forkert ND, Galinovic I, Rosenkranz M, Engelhorn T, Köhrmann M, Endres M, Kang DW, Dousset V, Sorensen AG, Liebeskind DS, Fiebach JB, Fiehler J, Gerloff C: DWI-FLAIR mismatch for the identification of patients with acute ischaemic stroke within $4.5 \mathrm{~h}$ of symptom onset (PRE-FLAIR): a multicentre observational study. Lancet Neurol 2011;10:978-986.

14 Butcher KS, Lee SB, Parsons MW, Allport L, Fink J, Tress B, Donnan G, Davis SM: Differential prognosis of isolated cortical swelling and hypoattenuation on CT in acute ischemic stroke. Stroke 2007;38:941-947.

15 Yoo AJ, Hu R, Hakimelahi R, Lev MH, Nogueira RG, Hirsch JA, González RG, Schaefer PW: CT angiography source images acquired with a fast-acquisition protocol overestimate infarct core on diffusion weighted images in acute ischemic stroke. J Neuroimaging 2011;17:1552-1569.

-16 Wintermark M, Flanders AE, Velthuis B, Meuli R, van Leeuwen M, Goldsher D, Pineda C, Serena J, van der Schaaf I, Waaijer A, Anderson J, Nesbit G, Gabriely I, Medina V, Quiles A, Pohlman S, Quist M, Schnyder P, Bogousslavsky J, Dillon WP, Pedraza S: Perfusion-CT assessment of infarct core and penumbra: receiver operating characteristic curve analysis in 130 patients suspected of acute hemispheric stroke. Stroke 2006;37:979-985.

17 Campbell BC, Christensen S, Levi CR, Desmond PM, Donnan GA, Davis SM, Parsons MW: Cerebral blood flow is the optimal CT perfusion parameter for assessing infarct core. Stroke 2011;42:3435-3440.

18 Yoo AJ, Pulli B, González RG: Imaging-based treatment selection for intravenous and intra-arterial stroke therapies: a comprehensive review. Expert Rev Cardiovasc Ther 2011;9:857-876.

19 Baker WL, Colby JA, Tongbram V, Talati R, Silverman IE, White CM, Kluger J, Coleman CI: Neurothrombectomy devices for the treatment of acute ischemic stroke: state of the evidence. Ann Intern Med 2011;154:243-252.

-20 Castaño C, Dorado L, Guerrero C, Millán M, Gomis M, Perez de la Ossa N, Castellanos M, García MR, Domenech S, Dávalos A: Mechanical thrombectomy with the Solitaire AB device in large artery occlusions of the anterior circulation: a pilot study. Stroke 2010;41:1836-1840.

21 Saver JL, Jahan R, Levy E, Jovin TG, Baxter B, Nogueira R, Clark W, Budzik R, Zaidat O0: Primary results of the Solitaire FR with the intention for thrombectomy (SWIFT) multicenter, randomized clinical trial. International Stroke Conference, New Orleans, La., USA, 2012.

-22 Abou-Chebl A: Endovascular treatment of acute ischemic stroke may be safely performed with no time window limit in appropriately selected patients. Stroke 2010;41:1996-2000.

23 Jovin TG, Liebeskind DS, Gupta R, Rymer M, Rai A, Zaidat 00, Abou-Chebl A, Baxter B, Levy EI, Barreto A, Nogueira RG: Imaging-based endovascular therapy for acute ischemic stroke due to proximal intracranial anterior circulation occlusion treated beyond 8 hours from time last seen well: retrospective multicenter analysis of 237 consecutive patients. Stroke 2011;42:2206-2211.

24 Hassan AE, Zacharatos H, Rodríguez GJ, Vazquez G, Miley JT, Tummala RP, Suri MF, Taylor RA, Qureshi AI: A comparison of computed tomography perfusion-guided and time-guided endovascular treatments for patients with acute ischemic stroke. Stroke 2010;41:1673-1678.

25 Mishra NK, Albers GW, Davis SM, Donnan GA, Furlan AJ, Hacke W, Lees KR: Mismatch-based delayed thrombolysis: a meta-analysis. Stroke 2010;41:e25-e33.

26 Lansberg MG, Straka M, Kemp S, Mlynash M, Wechsler LR, Joving TG, Wilder MJ, Lutsep HL, Czartoski TJ, Bernstein RA, Chang WJ, Warach S, Fazekas F, Manabu I, Tipirneni A, Hamilton SA, Zaharchuk G, Marks MP, Bammer R, Albers GW: MRI profile and response to endovascular reperfusion after stroke (DEFUSE 2): a prospective cohort study. Lancet Neurol 2012;11:860-867. 
27 Jovin TG, Yonas H, Gebel JM, Kanal E, Chang YF, Grahovac SZ, Goldstein S, Wechsler LR: The cortical ischemic core and not the consistently present penumbra is a determinant of clinical outcome in acute middle cerebral artery occlusion. Stroke 2003;34:2426-2433.

28 Warach S, Al-Rawi Y, Furlan AJ, Fiebach JB, Wintermark M, Lindstén A, Smyej J, Bharucha DB, Pedraza S, Rowley HA: Refinement of the magnetic resonance diffusion-perfusion mismatch concept for thrombolytic patient selection: insights from the desmoteplase in acute stroke trials. Stroke 2012;43:2313-2318.

29 Arenillas JF, Rovira A, Molina CA, Grive E, Montaner J, Alvarez-Sabin J: Prediction of early neurological deterioration using diffusion- and perfusion-weighted imaging in hyperacute middle cerebral artery ischemic stroke. Stroke 2002;33:2197-2203.

30 Yoo AJ, Verduzco LA, Schaefer PW, Hirsch JA, Rabinov JD, González RG: MRI-based selection for intra-arterial stroke therapy: value of pretreatment diffusion-weighted imaging lesion volume in selecting patients with acute stroke who will benefit from early recanalization. Stroke 2009;40:2046-2054. 